\title{
Neural Variability Quenching Predicts Individual Perceptual Abilities
}

\author{
Ayelet Arazi, ${ }^{1,2}$ Nitzan Censor, ${ }^{4}$ and Ilan Dinstein ${ }^{1,2,3}$ \\ ${ }^{1}$ Department of Brain and Cognitive Science, ${ }^{2}$ Zlotowski Center for Neuroscience, and ${ }^{3}$ Department of Psychology, Ben Gurion University of the Negev, \\ Beer-Sheva 8410501, Israel, and ${ }^{4}$ School of Psychological Sciences and Sagol School of Neuroscience, Tel Aviv University, Tel Aviv 6997801, Israel
}

Neural activity during repeated presentations of a sensory stimulus exhibits considerable trial-by-trial variability. Previous studies have reported that trial-by-trial neural variability is reduced (quenched) by the presentation of a stimulus. However, the functional significance and behavioral relevance of variability quenching and the potential physiological mechanisms that may drive it have been studied only rarely. Here, we recorded neural activity with EEG as subjects performed a two-interval forced-choice contrast discrimination task. Trial-by-trial neural variability was quenched by $\sim 40 \%$ after the presentation of the stimulus relative to the variability apparent before stimulus presentation, yet there were large differences in the magnitude of variability quenching across subjects. Individual magnitudes of quenching predicted individual discrimination capabilities such that subjects who exhibited larger quenching had smaller contrast discrimination thresholds and steeper psychometric function slopes. Furthermore, the magnitude of variability quenching was strongly correlated with a reduction in broadband EEG power after stimulus presentation. Our results suggest that neural variability quenching is achieved by reducing the amplitude of broadband neural oscillations after sensory input, which yields relatively more reproducible cortical activity across trials and enables superior perceptual abilities in individuals who quench more.

Key words: EEG; trial by trial neural variability; variability quenching; visual perception

\section{Significance Statement}

Variability quenching is a phenomenon in which neural variability across trials is reduced by the presentation of a stimulus. Although this phenomenon has been reported across a variety of animal and human studies, its functional significance and behavioral relevance have been examined only rarely. Here, we report novel empirical evidence from humans revealing that variability quenching differs dramatically across individual subjects and explains to a certain degree why some individuals exhibit better perceptual abilities than others. In addition, we found a strong relationship between variability quenching and suppression of broadband neural oscillations. Together, our results reveal the importance of reproducible cortical activity for enabling better perceptual abilities and suggest a potential underlying mechanism that may explain why variability quenching occurs.

\section{Introduction}

The mammalian brain is a remarkably variable system in which neural responses to repeated presentations of an identical stimulus exhibit considerable trial-by-trial variability (Arieli et al., 1996; Shadlen and Newsome, 1998; Carandini, 2004; Churchland et al., 2010; Goris et al., 2014). Neural variability can be separated

\footnotetext{
Received May 24, 2016; revised 0ct. 13, 2016; accepted 0ct. 19, 2016.

Author contributions: A.A., N.C., and I.D. designed research; A.A. performed research; A.A. analyzed data; A.A., N.C., and I.D. wrote the paper.

This work was supported by the generous support of the Israeli Science Foundation (Grant 961/14 to I.D.), the German Israeli Foundation (I.D.), the Berger Family (private donation to I.D.), and the I-CORE Israeli Science Foundation program (Grant $51 / 11$ to N.C.).

The authors declare no competing financial interests.

Correspondence should be addressed to Ayelet Arazi, Ben-Gurion University of the Negev, P.0.B. 653 Beer-Sheva, 8410501, Israel. E-mail: araziay@post.bgu.ac.il.

DOI:10.1523/JNEUROSCI.1671-16.2016

Copyright $\odot 2017$ the authors $\quad 0270-6474 / 17 / 370097-13 \$ 15.00 / 0$
}

into two distinct components: stimulus-evoked neural variability, which represents trial-by-trial variability in the amplitude and/or timing of the stimulus-evoked response, and ongoing neural variability, which represents spontaneous moment-bymoment fluctuations of neural activity. Previous electrophysiology studies have reported that stimulus-evoked variability (apparent after stimulus onset) is considerably smaller than ongoing neural variability (apparent before stimulus presentation) when measured with intracellular or extracellular recordings and regardless of whether the animal is performing a task, passively awake, or anesthetized (Monier et al., 2003; Finn et al., 2007; Mitchell et al., 2007; Churchland et al., 2010, 2011; Hussar and Pasternak, 2010; Ledberg et al., 2012; Qi and Constantinidis, 2012). Similar reductions in trial-by-trial neural variability after stimulus presentation have also been reported with electrocorticography (ECOG), fMRI, and magnetoencephalogram (MEG) recordings in humans (He and Zempel, 2013; 
He, 2013; Schurger et al., 2015). These studies reveal a general phenomenon, which is apparent at both the single-cell and neural network level, whereby stimulus-evoked responses quench ongoing neural variability.

What is the functional significance and behavioral relevance of neural variability quenching? A common assumption is that larger trial-by-trial neural variability should be detrimental for perceptual and cognitive performance (Dinstein et al., 2015; but also see Garrett et al., 2013b). An intuitive explanation for this can be found in signal detection theory (Green and Swets, 1966), which suggests that internal noise is detrimental for the sensitivity of a detection system. Previous studies have estimated internal noise levels in different sensory domains using behavioral techniques such as the equivalent noise model (Pelli and Farell, 1999), the double-pass method (Burgess and Colborne, 1988; Neri, 2010), or by examining the slope of the psychometric function (shallower slopes are thought to indicate a "noisier" system; Buss et al., 2009; Jones et al., 2014). Such studies have reported that individuals with higher levels of internal noise exhibit higher discrimination thresholds (Legge et al., 1987; Pardhan, 2004) and have inferred that internal noise limits the perceptual abilities of an individual. But behavioral studies cannot quantify ongoing neural variability and differentiate it from stimulus evoked neural variability. Therefore, these studies cannot determine which form of neural variability/noise (absolute neural variability before/after stimulus presentation or the extent of variability quenching) is relevant for perceptual performance.

Neural variability across trials is a direct neurophysiological measure of internal noise. In agreement with the behavioral studies described above, several neuroimaging studies have reported that variability is lower across trials in which a threshold-level visual stimulus is consciously perceived/detected (Schurger et al., 2010, 2015), as well as across trials in which a stimulus is later remembered (Xue et al., 2010). Although these seminal studies have provided critical evidence demonstrating that neural variability is indeed detrimental for accurate perception and memory in humans, only one of them (Schurger et al., 2015) differentiated between ongoing and stimulus evoked neural variability and quantified variability quenching. This point is particularly important given that others in the field have suggested that temporal variability (i.e., fluctuations over time, as measured by the variability of fMRI time courses) is beneficial for cognitive performance (Garrett et al., 2011, 2014). Elucidating the behavioral relevance of distinct types of neural variability and revealing their potential neurophysiological sources is therefore of great interest.

To address these questions, we quantified trial-by-trial neural variability in individual subjects using EEG while participants performed a contrast discrimination task. We examined neural variability before and after stimulus presentation and quantified the extent to which neural variability was quenched in each individual. Using spectral analyses, we also investigated whether neural variability quenching was associated with decreased EEG power or increased phase coherence across trials, two measures that represent alternative mechanisms that govern trial-by-trial variability. This enabled us to determine which forms of neural variability explained individual perceptual abilities and gain insight into their potential underlying neurophysiological mechanisms.

\section{Materials and Methods}

\section{Subjects}

Twenty-two subjects ( 7 males, mean age $25 \pm 2.7 \mathrm{SD}$ ) participated in the study. All subjects had normal or corrected-to-normal vision. The study was approved by the Ben-Gurion University Internal Review Board. Subjects provided written informed consent and were either paid for their participation (\$12 US per hour) or received research credit as part of the Psychology Department undergraduate program.

\section{Stimuli and experimental design}

The experiment was performed in a dark and sound proof room. The stimuli were presented using MATLAB (The MathWorks) and Psychtoolbox (Brainard, 1997). The stimulus consisted of a circular checkerboard with a diameter of $3.7^{\circ}$ visual angle located at the center of the screen. Stimuli were presented using a CRT monitor with a $60 \mathrm{~Hz}$ refresh rate and a resolution of $1280 \times 1024$ pixels.

A two-interval-forced-choice (2IFC) procedure was used to measure trial-by-trial neural variability and estimate contrast discrimination thresholds simultaneously (Fig. 1A). Each trial consisted of two stimuli that were presented sequentially: one interval contained a stimulus at $100 \%$ contrast (i.e., base contrast, $C_{\mathrm{b}}$ ) and the other interval contained a stimulus at $100 \%$ contrast minus a varying target contrast $\left(C_{\mathrm{t}}\right)$, such that the final contrast was $C_{\mathrm{b}}-C_{\mathrm{t}}$. The order of stimuli was randomized. Each stimulus was presented for $100 \mathrm{~ms}$, followed by a $900 \mathrm{~ms}$ blank screen. Subjects were then presented with a white fixation cross at the center of the screen until they reported which of the stimuli had higher contrast. Auditory feedback $(1000 \mathrm{~Hz}$; stimulus duration $50 \mathrm{~ms}$; stimulus intensity $60 \mathrm{db}$ ) was given only after a correct response. The fixation cross remained on the screen for an additional $900 \mathrm{~ms}$ (intertrial interval) and then the subsequent trial began.

We chose this 2IFC rather than a two-alternative force choice (2AFC) task because its structure enabled us to easily extract the neural responses to trials containing the base stimulus ( $100 \%$ contrast), which was presented on every trial in either the first or second stimulus interval. We replicated our results across the two intervals to demonstrate that the results were not specifically associated with short-term memory requirements that are likely to exist in the first interval of the 2IFC task. Note that a 2AFC task would not work as well here because we would have to randomize the location of the low-contrast stimulus (right or left) across trials and present it together with a second stimulus of varying contrast. This would dramatically reduce the number of trials available for the variability analysis that was performed only across trials containing an identical stimulus.

Individual discrimination thresholds were initially estimated using a two-down, one-up staircase procedure to estimate the contrast discrimination threshold of each subject at a level of $70.7 \%$ correctness (Levitt, 1970). The initial target contrast was $C_{\mathrm{t}}=80 \%$ (i.e., stimulus contrast $=$ $20 \%$ ). The target contrast was divided by 2 or $\sqrt{2}$ after two correct responses and multiplied by 2 or $\sqrt{ } 2$ after one incorrect response ( $\sqrt{ } 2$ was used after the third reversal; Hawkey et al., 2004). The procedure was terminated after 12 reversal (mean number of trials: $79 \pm 9$ SD) and the geometric mean of the last 8 reversal values was used as the threshold estimation.

Next, we estimated the psychometric function for each individual using the same 2IFC task with two target contrasts above and two below the previously estimated threshold. Subjects completed four blocks containing 100 trials each, 20 trials per contrast level presented in a random order for a total of 80 trials per contrast level. To ensure that the chosen contrast levels covered the entire range of the psychometric function, a function was estimated after the first block of trials and the range was adjusted appropriately. The final psychometric function, discrimination threshold, and slope were estimated as described below.

\section{EEG and eye tracking recordings}

EEG data were recorded using a 64-channel Bio-Semi system. Data were referenced to the mastoid electrodes. Electrooculography was recorded using two electrodes at the outer canthi of the left and right eyes and one electrode placed below the right eye. In addition, the position of the right eye was recorded simultaneously with an eye tracker at $1000 \mathrm{~Hz}$ (EyeLink 1000; SR Research).

\section{EEG preprocessing}

Data were analyzed using MATLAB and the EEGLAB toolbox (Delorme and Makeig, 2004). Continuous EEG data were down-sampled to $512 \mathrm{~Hz}$ 
A

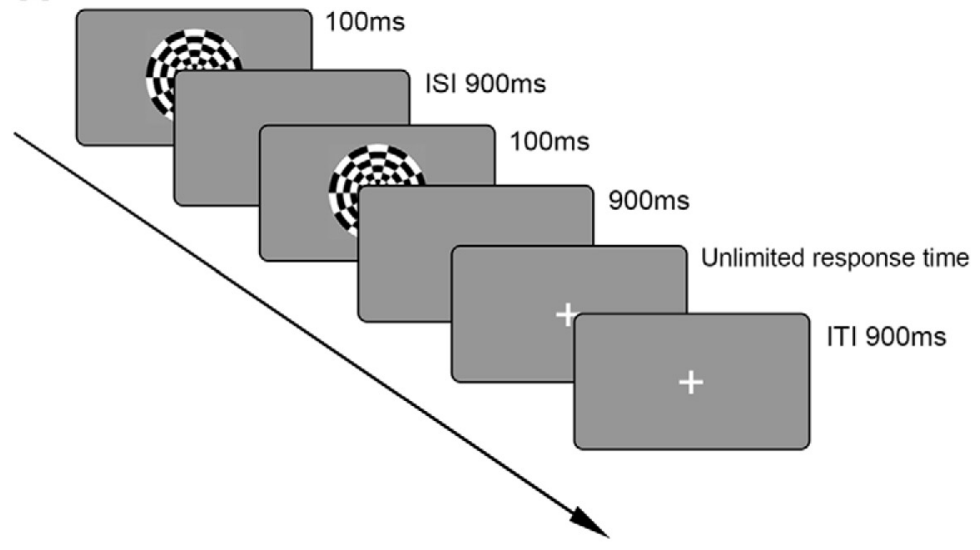

B

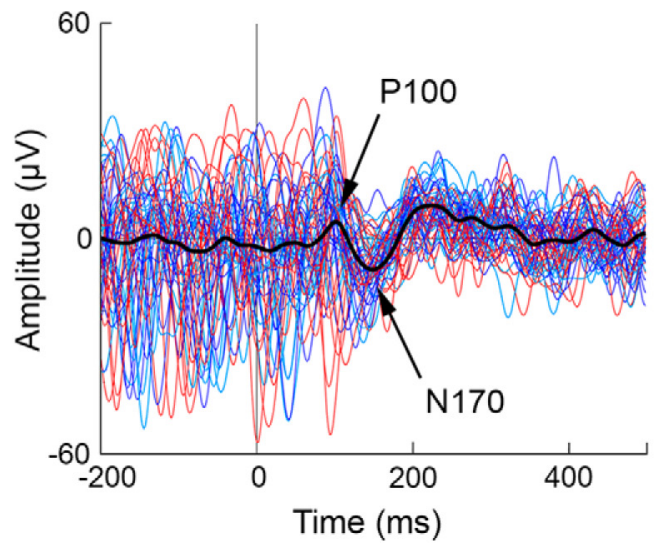

Figure 1. Experimental design and EEG responses. A,2IFC contrast discrimination task included the presentation of two consecutive stimuli. Each stimulus was presented for $100 \mathrm{~ms}$ and was followed by a $900 \mathrm{~ms}$ blank screen. Subjects were instructed to press a button to report which stimulus was of higher contrast when the fixation cross appeared. One of the two stimuli was always at base contrast (100\%) and the other was of lower contrast. B, EEG recordings from single trials containing the base stimulus. Colored lines represent single trials and the black line represents the mean ERP. This example presents trials from the first stimulus interval of a single subject (electrode P08).

\section{First stimulus interval}

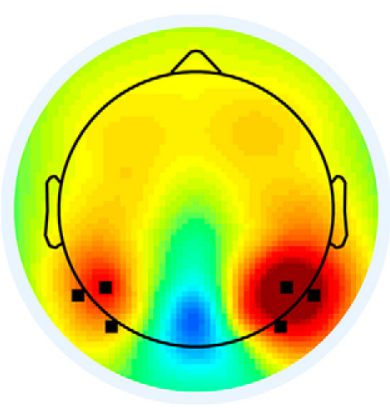

\section{Second stimulus interval}
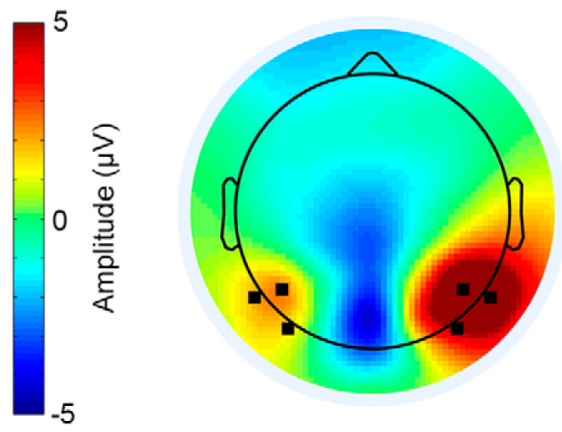

Figure 2. Visual system responses. Topographic maps (mean across subjects) of the P100 response in the first (left) and second (right) stimulus intervals. We selected the electrodes marked in black (P6, P8, P08, P5, P7, and P07) for further analyses.

and filtered using a $1-40 \mathrm{~Hz}$ band-pass filter. EEG epochs were extracted using a time window of $700 \mathrm{~ms}$ ( $200 \mathrm{~ms}$ prestimulus to $500 \mathrm{~ms}$ poststimulus). Only base trials containing a stimulus contrast of $100 \%$ were examined in the neural variability analyses and baseline correction was not performed so as not to alter trial-by-trial variability in the prestimulus interval.

Trials in which the absolute amplitude exceeded $70 \mu \mathrm{V}$ or the power exceeded $25 \mathrm{db}$ in the $20-40 \mathrm{~Hz}$ frequency range were identified as containing eye blinks or muscle artifacts, respectively, and were removed from further analysis. Identification of eye blinks was confirmed by eye tracking. Trials containing horizontal or vertical eye movements that exceeded 1.5 SDs of the mean were identified and excluded from all analyses. Mean number of base trials per subject after rejection was $159 \pm 28$ SD trials.

The cleaned and segmented EEG data were decomposed into independent components (IC) using the runica function as implemented in EEGLAB. Components representing eye movements or muscle contractions were excluded by visual inspection. We computed the percentage variance explained by each of the remaining components within a time window of 50-300 ms after the stimulus, corresponding to the typical duration of a visually evoked response. We then chose the ICs that accounted for at least $75 \%$ of the variance within this time window and thereby maximally captured the neural responses associated with visual processing in each subject (Makeig et al., 1997). Further analyses were performed twice, first with the cleaned EEG data and again using the selected ICs. This enabled us to determine whether conclusions regarding neural variability measures were equally evident when extracting all ICs that were not related to visual system responses (i.e., potentially containing non-neural sources of variability such as anecdotal head movements or multiple forms of measurement noise).

\section{EEG data analysis}

Time domain analysis. Trial-by-trial variability was computed for each time point in the extracted epochs ( -200 to $500 \mathrm{~ms}$ ) for each of the 64 electrodes in each subject separately. Base trials from the first and second stimulus intervals were analyzed separately. Absolute trialby-trial variability in the prestimulus $\left(\operatorname{Var}_{\text {pre }}\right)$ and poststimulus $\left(\operatorname{Var}_{\text {post }}\right)$ stimulus intervals was computed as the mean variance in -200 to $0 \mathrm{~ms}$ and 150 to $400 \mathrm{~ms}$ time windows, respectively. Relative trial-by-trial variability (i.e., quenching level) was estimated for each subject using three alternative measures: (1) by computing the mean variability in the poststimulus interval in percentage change units as follows: relative variance $=\left(\frac{V a r_{\text {post }}}{V a r_{\text {pre }}}-1\right) * 100 ;(2)$ by computing the absolute difference between prestimulus variability and poststimulus variability as follows: relative variance $=$ $V a r_{\text {pre }}-V a r_{\text {post }}$; and (3) by computing the ratio between prestimulus variability and poststimulus variability as follows: relative variance $=\frac{V a r_{\text {pre }}}{V a r_{\text {post }}}$ (see Fig. 5). We focused our analyses on the six electrodes (P6, P8, PO8, P5, P7, and PO7) that exhibited the strongest visual P100 responses (Fig. 2). We identified the amplitude of the P100 response for each subject and in each electrode separately and then averaged across subjects.

Mean EEG activity. We quantified mean EEG activity across the six occipital electrodes during the same time period that contained stable variability quenching (i.e., $150-400 \mathrm{~ms}$ after stimulus presentation). We calculated the mean event-related potential (ERP) across trials in each electrode separately, computed the area under the rectified 
A First stimulus interval

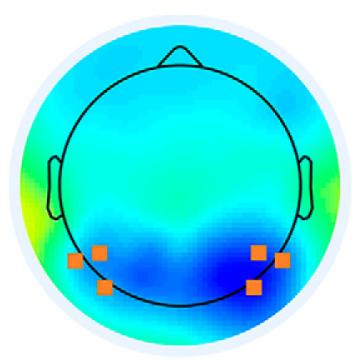

Second stimulus interval

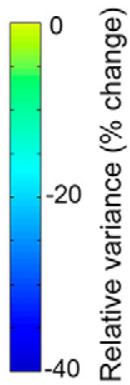

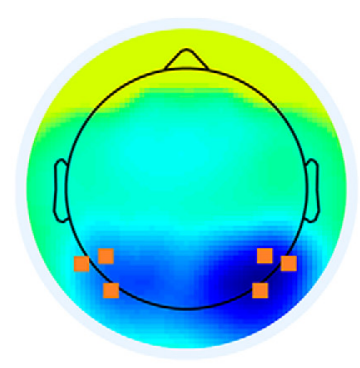

B
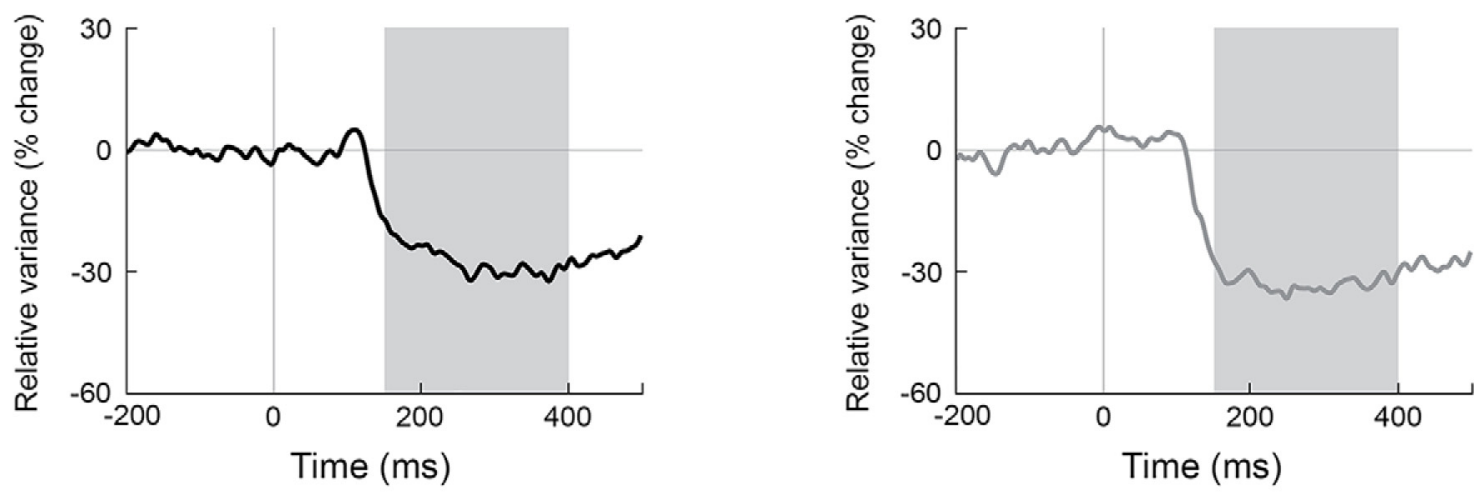

C

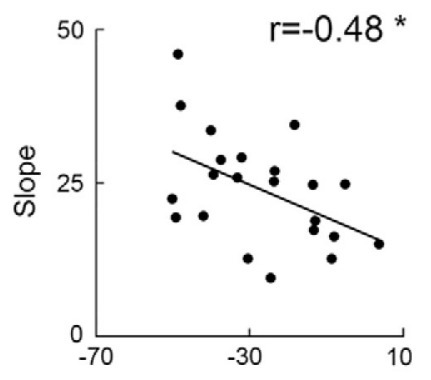

Relative variance (\% change)

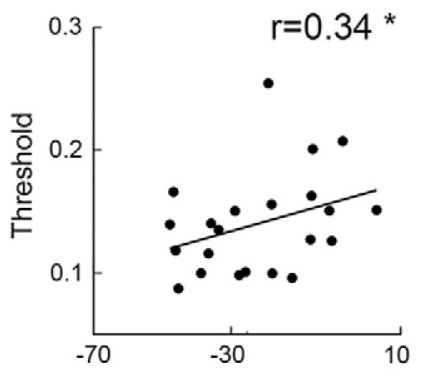

Relative variance (\% change)

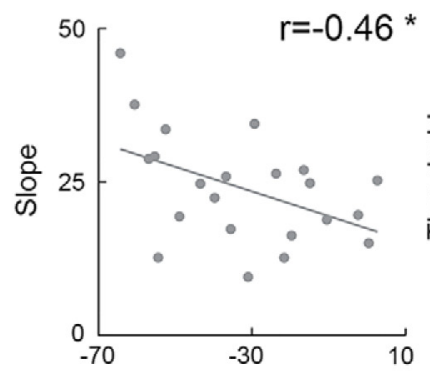

Relative variance (\% change)

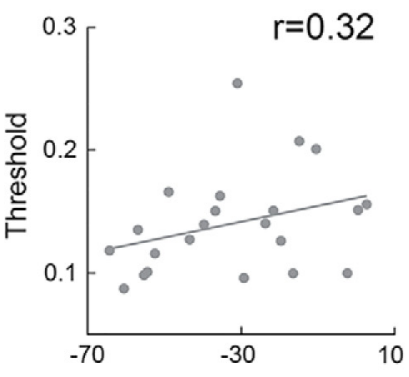

Relative variance (\% change)

Figure 3. Neural variability quenching predicts perceptual performance. Neural variability quenching was estimated in the first (left) and second (right) stimulus intervals. $\boldsymbol{A}$, Topographic maps (mean across subjects) representing the level of variability quenching 150 - $400 \mathrm{~ms}$ after stimulus presentation. $\boldsymbol{B}$, Time courses of trial-by-trial variability in percentage change units demonstrating the decrease in neural variability after stimulus presentation (mean across electrodes noted in $A$ and across subjects). Because neural variability decreased and remained stable between 150 and 400 ms after stimulus presentation (marked in gray), we estimated the level of variability quenching for each subject as the mean across this time window. $\boldsymbol{C}$, Correlations between relative neural variability (quenching) and psychometric function slope (left) or contrast discrimination thresholds (right). Each dot represents a single subject. Asterisks indicate significant correlations as assessed by a randomization test $(p<0.05)$.

curve (integral), and then computed the mean across the six electrodes.

Time-frequency analysis. Each trial/epoch was transformed to the time-frequency domain using a sliding window fast Fourier transform analysis. The Fourier coefficients were estimated in windows of 64 samples (corresponding to $125 \mathrm{~ms}$ ) that were shifted by 4 samples ( $8 \mathrm{~ms}$ ) along the time domain. The power of each time-frequency point was obtained by computing the square root of sum of squares of the Fourier coefficients. Mean power across trials was calculated for the theta- $(4-7$ $\mathrm{Hz})$, alpha- $(8-13 \mathrm{~Hz})$, beta- $(14-20 \mathrm{~Hz})$, and low-gamma $(25-40 \mathrm{~Hz})-$ frequency bands and then normalized to percentage change units with respect to the prestimulus interval $(-200$ to $0 \mathrm{~ms})$ : relative power $=$ $\left(\frac{\text { Power }_{\text {post }}}{\text { Power }_{\text {pre }}}-1\right) * 100$. Intertrial phase coherence (ITPC), a measure of the degree to which the phase of each frequency is aligned across trials, was also calculated for each of the frequency bands (Delorme and Makeig, 2004). Responses with similar timing/latencies across trials are expected to have stronger phase coherence than responses with variable timing.

\section{Psychometric function estimation}

We fitted a psychometric function to the behavioral data of each subject using a maximum likelihood method as implemented in Palameds toolbox (Prins and Kingdom, 2009) and then used the following logistic function:

$$
F(x ; \alpha, \beta)=\frac{1}{1+\exp (-\beta(x-\alpha))}
$$

where $\alpha$ is the threshold, $\beta$ is the slope and $x$ is the contrast difference between the two intervals, $C_{\mathrm{b}}-C_{\mathrm{t}}$. The lapse rate was set to 0.01 and 
A

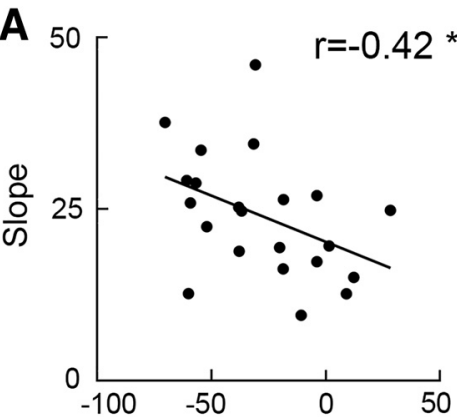

Relative variance (\% change)

C

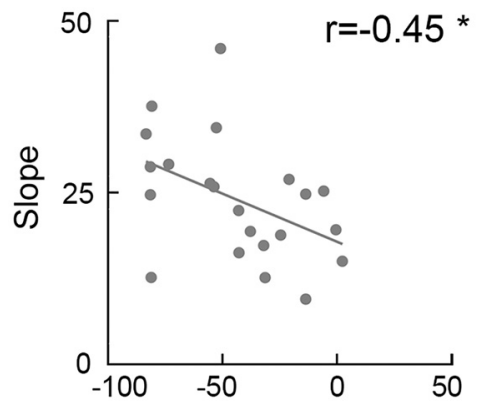

Relative variance (\% change)
B

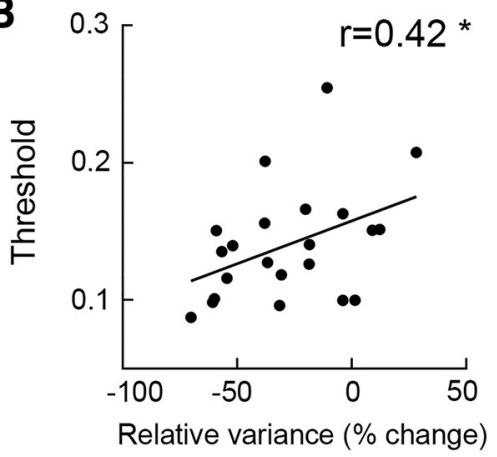

D

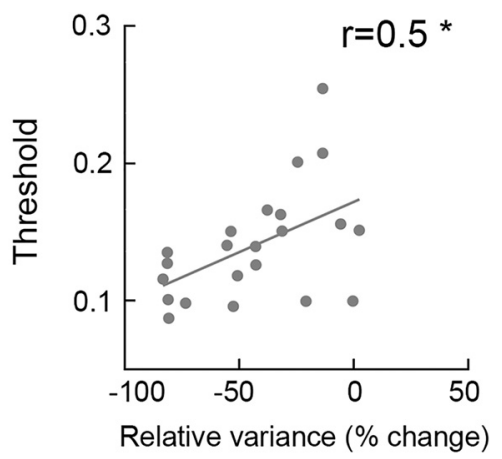

Figure 4. Neural variability quenching predicts perceptual performance also when examining the EEG data after extracting the visual ICA components (see Materials and Methods). Correlations between variability quenching as estimated in the first $(\boldsymbol{A}, \boldsymbol{B})$ and second $(\boldsymbol{C}, \boldsymbol{D})$ stimulus intervals and psychometric function measures (slope and threshold). Each dot represents a single subject. Asterisks indicate significant correlations assessed by a randomization test $(p<0.05)$.

\section{A} Absolute difference between pre and post stimulus variability

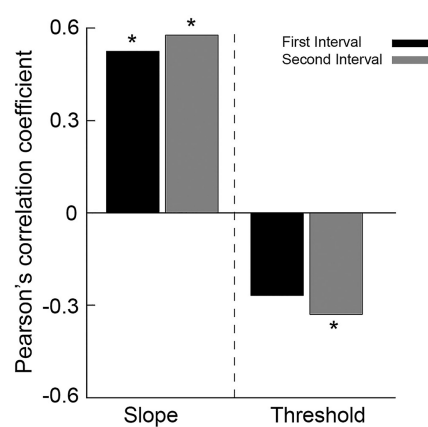

B Ratio between pre and post stimulus variability

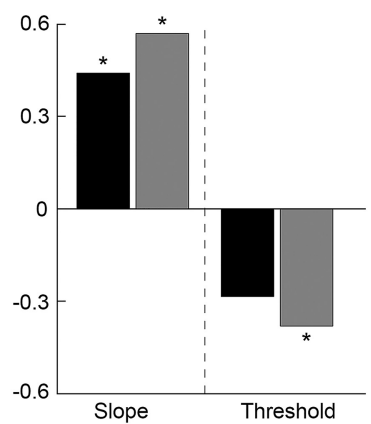

Figure 5. Alternative measures of variability quenching explain individual perceptual performance in a similar manner demonstrating the robustness of this relationship. Correlation coefficients between individual magnitudes of variability quenching and perceptual performance estimates (slope and threshold) when using absolute difference between neural variability in the pre ( -200 to $0 \mathrm{~ms})$ and post $(150-400 \mathrm{~ms})$ stimulus intervals $(\boldsymbol{A})$ and the ratio between neural variability in the prestimulus ( -200 to $0 \mathrm{~ms}$ ) and poststimulus ( $150-400 \mathrm{~ms}$ ) intervals $(\boldsymbol{B})$. Asterisks indicate significant correlations assessed by a randomization test $(p<$ $0.05)$.

guess rate to 0.5 (i.e., at chance level). The discrimination threshold ( $80 \%$ correct level) and psychometric function slope were extracted for each subject separately.

We used the "deviance" measure to estimate the goodness-of-fit of the psychometric functions in each subject (Wichmann and Hill, 2001). Deviance is the ratio between the log likelihood function of a saturated model, in which the number of parameters equals the number of data points and the psychometric function containing two free parameters. Deviance values can range from zero to infinity with very low deviance values indicating that the psychometric function is close to a perfect fit with the data (i.e., likely to be an overfit) and very large values of deviance indicating that there are large errors/residuals when fitting the model to the data. One way of quantifying the goodness-of-fit of each subject is to determine whether the fit of each subject falls between the $5^{\text {th }}$ and $95^{\text {th }}$ percentile of a parametric bootstrapped distribution generated by randomly sampling 5000 datasets using the psychometric function that was fit to the data as a generating function. For the true fit to be considered "good," its deviance had to be between the $5^{\text {th }}$ and $95^{\text {th }}$ percentile of this distribution of deviances (Wichmann and Hill, 2001).

\section{Statistical tests}

We examined the relationship between neural (EEG) and perceptual measures across subjects using Pearson's and Spearman's correlations. The statistical significance of the correlation coefficients was assessed with a permutation/ randomization test in which we shuffled the labels of the subjects before computing the correlation coefficient. This procedure was performed 10,000 times while shuffling the labels across subjects randomly each time to generate a null distribution for each pair of EEG and perceptual measures (e.g., prestimulus neural variability and discrimination threshold). For the true correlation coefficient to be considered significant, it had to be higher than the $95^{\text {th }}$ percentile or lower than the $5^{\text {th }}$ percentile of this null distribution (i.e., equivalent to a $p$-value of 0.05 in a one tailed $t$ test).

An important feature of our study was that we were able to reproduce our results across neural responses in the first and second stimulus intervals. In other words, the relationship between variability quenching and perceptual performance was reproduced in two independent assessments. To quantify the statistical significance of this reproducibility, we computed the Bayes factor, which quantifies the evidence for the null hypothesis compared with an alternative hypothesis given a prior. Conventionally, a Bayes factor that is $>3$ offers strong support for the alternative hypothesis and one lower than $1 / 3$ provides strong support for the null hypothesis (Dienes, 2014). To quantify the reproducibility of the correlation between the psychometric function slope/threshold and variability quenching, we tested whether there was evidence of a relationship in the second stimulus interval given the correlation values found in the first stimulus interval (i.e., the prior; Wagenmakers et al., 2016).

\section{Gaze variability}

Relative gaze position was measured for each time point as the distance from the fixation cross. We then calculated the SD in gaze position across trials for each time point and computed the mean across time points in the poststimulus interval ( 0 to $500 \mathrm{~ms}$ ). Individuals who move their eyes more exhibit larger trial-by-trial SD in gaze position than individuals who move their eyes less. Therefore, we used this measure to determine whether gaze variability could explain individual neural variability measures (see Fig. 11A). Two participants were excluded from this analysis due to difficulties in the calibration process of the eye tracker.

\section{Electrode offset variability}

The quality of EEG recordings was estimated by computing electrode offset variability across trials. The mean offset value was first computed for each trial, the variability across trials was computed for each of the four electrodes, and finally the variability was averaged across electrodes (see Fig. 11B). When using active electrode, there is no measure of impedance, and fluctuations in electrode offset are the best indication of the quality of electrode recording (Kappenman and Luck, 2010). 
A First stimulus interval

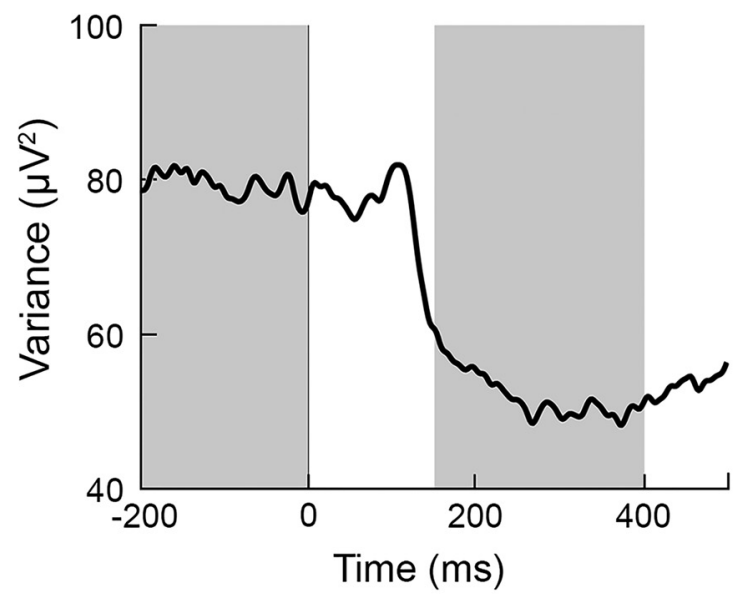

B

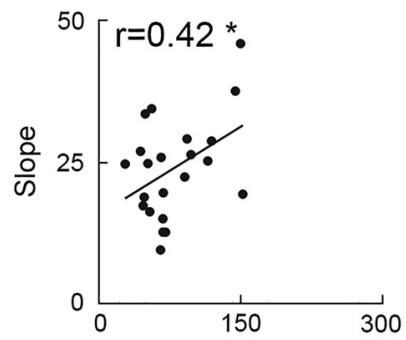

Pre stimulus variability

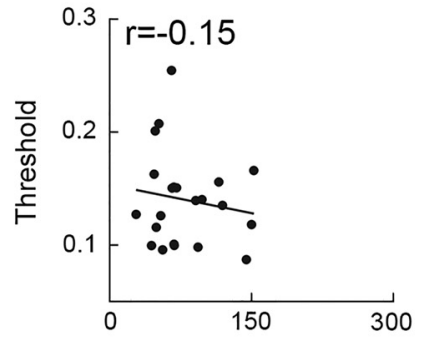

Pre stimulus variability

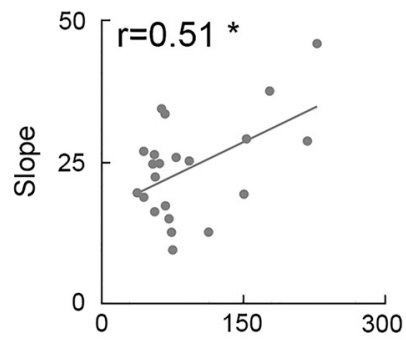

Pre stimulus variability

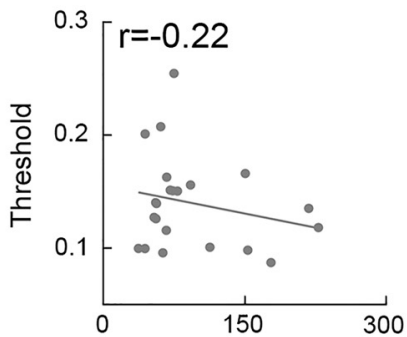

Pre stimulus variability

C

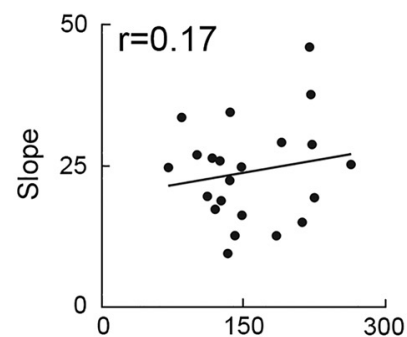

post stimulus variability

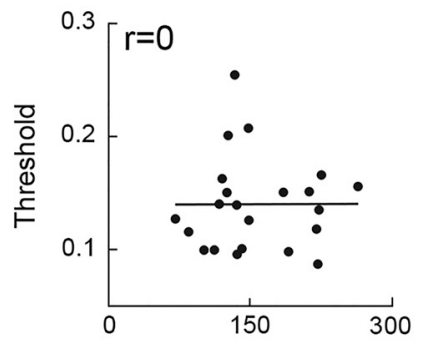

post stimulus variability

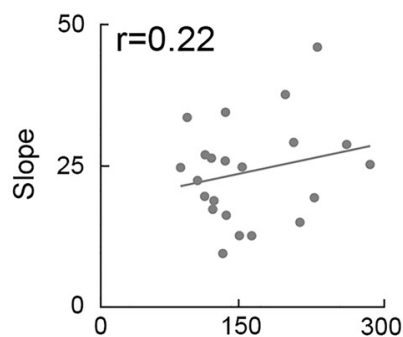

post stimulus variability

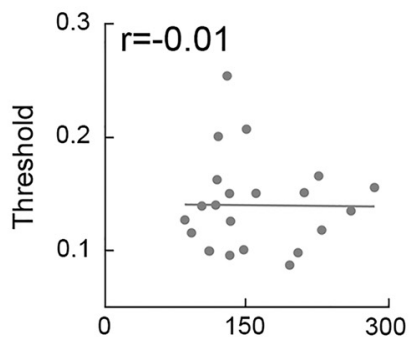

post stimulus variability

Figure 6. Absolute neural variability in the prestimulus interval, but not the poststimulus interval, predicts perceptual performance. $\boldsymbol{A}$, Time courses of absolute trial-by-trial variability in the first (left) and second (right) stimulus intervals. $\boldsymbol{B}$, Correlations between perceptual measures (slope and threshold) and prestimulus variability. $\boldsymbol{C}$, Correlations between perceptual measures (slope and threshold) and poststimulus variability. Each dot represents a single subject. Asterisks indicate significant correlations as assessed by a randomization analysis $(p<0.05)$.

\section{Results}

Individual subjects exhibited typical ERP responses to the presentation of the high-contrast checkerboard stimulus (base trials with $100 \%$ contrast), which included positive and negative peaks at 100 and $170 \mathrm{~ms}$ after stimulus presentation, respectively (Fig. $1 B$ ). Note that the responses varied considerably across trials and there was a clear decrease in variability $\sim 150 \mathrm{~ms}$ after stimulus presentation. Because we wanted to focus on visual system responses, we selected the six electrodes with the strongest $\mathrm{P} 100$ responses (P6, P8, PO8, P5, P7, and PO7) for further analyses (Fig. 2).

\section{Neural variability and measurement noise}

When estimating trial-by-trial neural variability in single subjects, it is important to ensure that estimates are not biased by measurement noise that may differ across subjects. Therefore, we used automated software to identify and exclude all trials containing eye blinks, muscle contractions, and saccades (see Materials and Methods). In addition, we performed an IC analysis (ICA) and selected the components that explained $75 \%$ of the variance in the stimulus evoked response period (50-300 ms after stimulus onset). In this manner, we extracted the spatiotemporal components of the data that best captured visual system responses in each subject while removing all other components, which may have contained variability due to non-neural sources (Makeig et al., 1997). All of the analyses described below were performed once with the original cleaned EEG recordings and again after selection of the relevant ICA components in each subject. 

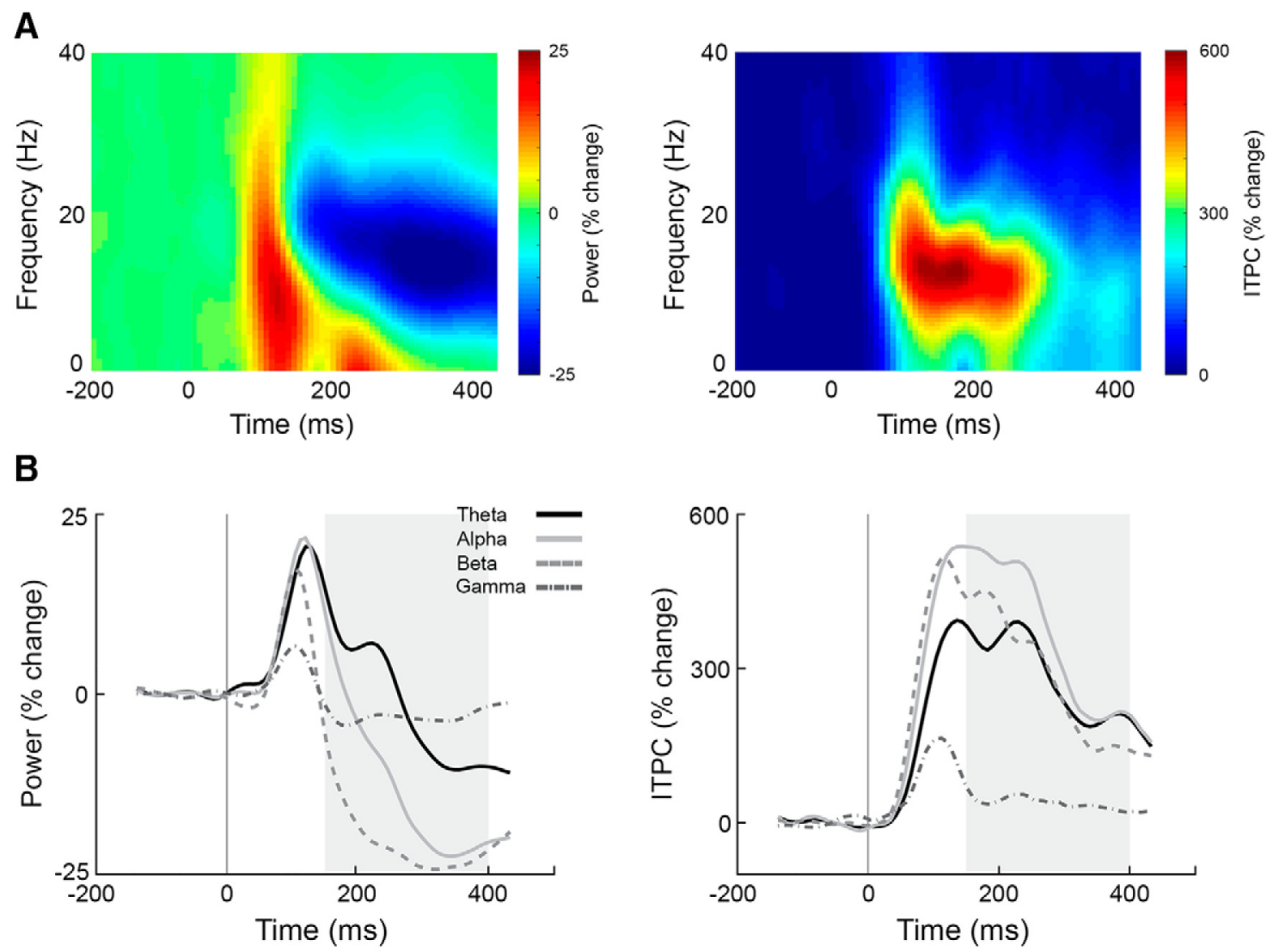

Figure 7. Spectral power and ITPC dynamics (mean across all subjects). $\boldsymbol{A}$, Time-frequency spectrograms of the spectral power (left) and ITPC (right) demonstrating the change in power and phase coherence as a function of time, with color representing the amplitude of change relative to the prestimulus interval ( -200 to $0 \mathrm{~ms})$ in percentage change units. $\boldsymbol{B}$, Time courses of four frequency bands: theta ( $4-7 \mathrm{~Hz}$; black lines), alpha $(8-13 \mathrm{~Hz}$; light gray lines), beta ( $14-20 \mathrm{~Hz}$; light gray dotted lines), and low-gamma ( $25-40 \mathrm{~Hz}$; dark gray dotted line). Area marked in gray $(150-400 \mathrm{~ms})$ corresponds to time window with sustained neural variability quenching in Figure 3.

A

Theta $(4-8 \mathrm{~Hz})$

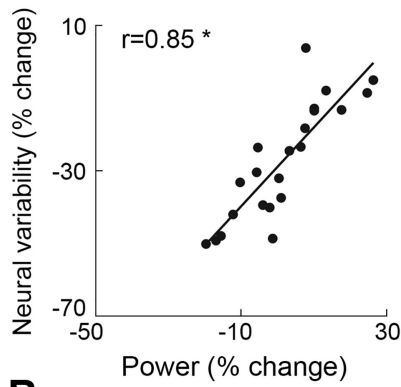

B

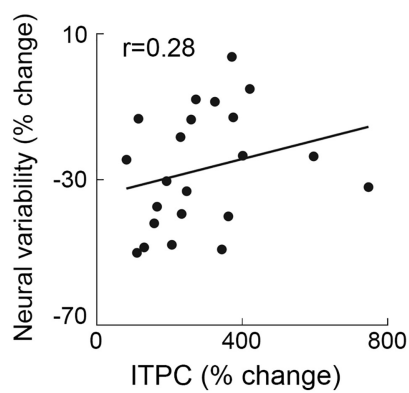

Alpha $(8-13 \mathrm{~Hz})$
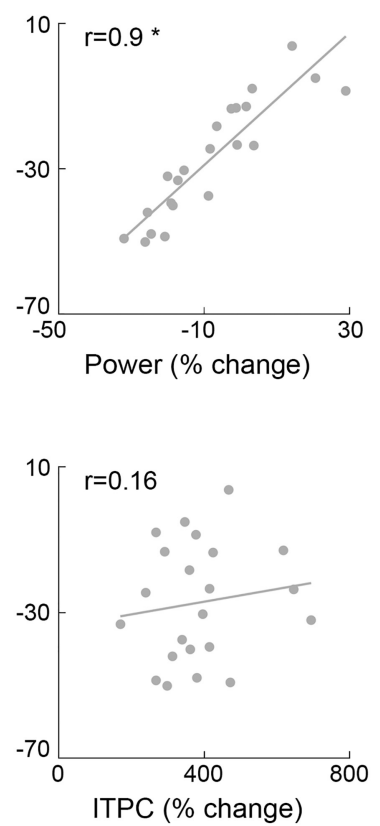

Beta $(13-20 \mathrm{~Hz})$

Gamma $(25-40 \mathrm{~Hz})$
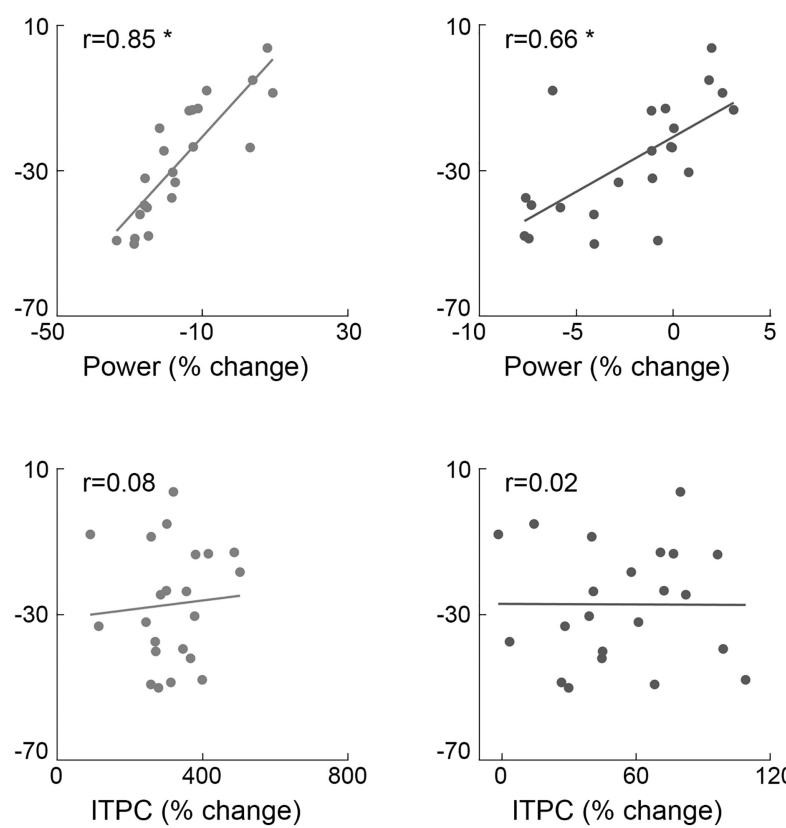

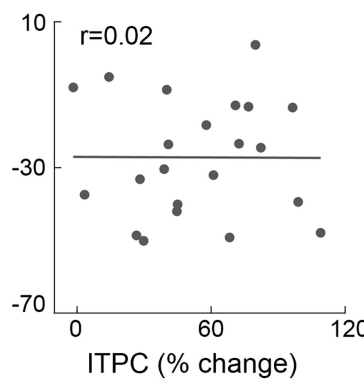

Figure 8. Variability quenching is related to decreased power but not to increased ITPC. $A$, Scatter plots demonstrating the relationship between neural variability quenching and spectral power. $B$, Scatter plots demonstrating the relationship between neural variability quenching and ITPC. Correlations were assessed for four frequency bands: theta $(4-7 \mathrm{~Hz})$, alpha $(8-13 \mathrm{~Hz}), \mathrm{beta}(14-20$ $\mathrm{Hz})$, and low-gamma $(25-40 \mathrm{~Hz})$. Each dot represents single subjects. Asterisks indicate significant correlations as assessed by a randomization analysis $(p<0.05)$. 

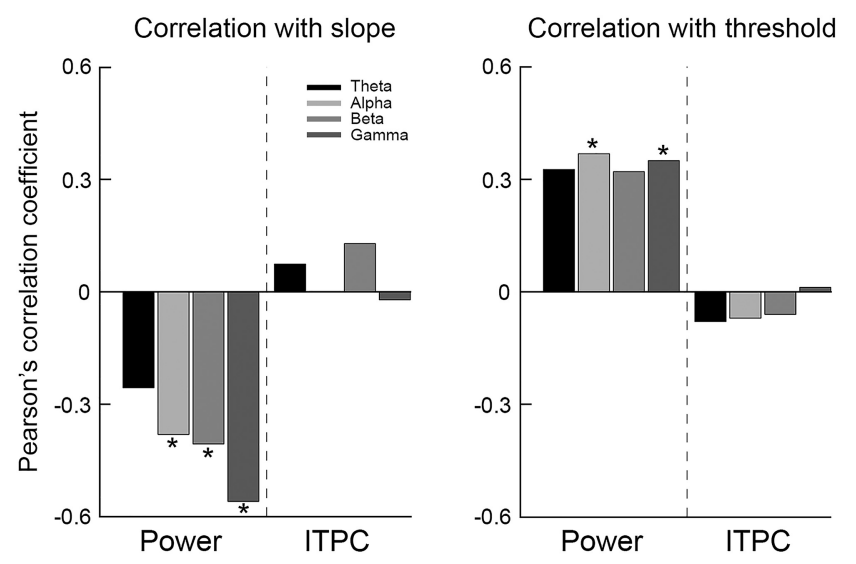

Figure 9. Better perceptual performance is associated with decreased power rather than increased ITPC. Pearson's correlation coefficients describing the relationships between psychometric function slope (left) or discrimination threshold (right) and spectral power or ITPC as estimated in four frequency bands: theta (black), alpha (light gray) beta (gray), and lowgamma (dark gray). Asterisks indicate significant correlations as assessed by a randomization analysis $(p<0.05)$.

\section{Neural variability quenching}

In agreement with previous studies, our subjects exhibited a strong and sustained decrease in variability across trials $150-400$ $\mathrm{ms}$ after stimulus presentation. This finding was robust in both the first and second stimulus intervals (Fig. $3 B$ ) and strongest in occipital electrodes (Fig. 3A).

Individual variability quenching magnitudes predicted perceptual abilities (Fig. 3C). Significant negative correlations were apparent between the slope of the psychometric function and the magnitude of neural variability quenching, as estimated in either the first or second stimulus intervals (Pearson's: $r_{(22)}<-0.46$, $p<0.05$; Spearman's: $r_{(22)}<-0.44, p<0.05$; randomization analysis). Significant positive correlations were revealed between individual discrimination thresholds and the level of neural variability quenching as estimated in the first stimulus interval (Pearson's: $r_{(22)}=0.34, p<0.05$; Spearman's: $\left.r_{(22)}=0.33, p<0.05\right)$ and in the second stimulus interval, when we used the Spearman's correlation coefficient $\left(r_{(22)}=0.4, p<0.05\right)$. Strong trend in the same direction was also apparent when we used Pearson's correlation coefficient $\left(r_{(22)}=0.32, p=0.07\right)$.

We computed the Bayes factor to quantify the reproducibility of the correlation analyses between the perceptual measures and the magnitude of variability quenching across the two stimulus intervals. We found strong evidence for this relationship/correlation when testing the correlation coefficient found in the second stimulus interval, given the correlation coefficient in the first stimulus interval as a prior (Bayes factor for the correlation with the threshold was 3.9 and for the correlation with the slope was 6.2). Equivalent findings were also found when examining the visual ICA components (Fig. 4) and two alternative quenching measures (Fig. 5), thereby demonstrating the robustness of these results across multiple manners of analysis.

The relationship between individual variability quenching and discrimination performance was specific to the examined electrodes. Performing the same analysis with frontal electrodes (FP1, FP2 and FPZ) revealed low correlation with discrimination threshold (first stimulus interval: $r_{(22)}=0.27$, second stimulus interval: $\left.r_{(22)}=0.09 ; p>0.11\right)$ and with the slope of the psychometric function (first stimulus interval: $r_{(22)}=-0.32, p=$ 0.7 ; second stimulus interval: $\left.r_{(22)}=-0.25, p=0.13\right)$.

\section{Pre and post stimulus neural variability}

Neural variability quenching represents a relative difference between the neural variability apparent before and after stimulus presentation. We also examined the relationship between perceptual performance and the absolute pre and post stimulus neural variability levels of individual subjects. Significant positive correlations were found between the psychometric function slope and absolute neural variability in the prestimulus interval $(-200$ to 0 $\mathrm{ms}$ ) as quantified in either the first or second stimulus intervals using the Pearson's correlation coefficient (first interval $r_{(22)}=$ 0.42 , second interval $r_{(22)}=0.51, p<0.05$ ) but not when using the Spearman correlation coefficient (first interval $r_{(22)}=0.25$, second interval $r_{(22)}=0.23, p<0.12$; Fig. $6 B$ ). Weak, nonsignificant negative correlations were found between discrimination thresholds and neural variability in the prestimulus period (Pearson's: first interval $r_{(22)}=-0.15$, second interval $r_{(22)}=-0.22$, $p>0.16$; Spearman's: first interval $r_{(22)}=-0.1$, second interval $\left.r_{(22)}=-0.04, p>0.33\right)$. There were no significant correlations between either psychometric function slopes (Pearson's: first interval $r_{(22)}=0.17$, second interval $r_{(22)}=0.22, p>0.15$; Spearman's: first interval $r_{(22)}=0.06$, second interval $r_{(22)}=0.1 p>$ 0.32 ) or discrimination thresholds (Pearson's: first interval $r_{(22)}$ $=0$, second interval $r_{(22)}=-0.01, p>0.48$; Spearman's: first and second intervals $\left.r_{(22)}=0.1, p=0.3\right)$ and neural variability in the poststimulus interval (Fig. $6 C$ ).

\section{Variability in the amplitude and the latency of the neural responses}

Stimulus-evoked neural variability can be driven by variability in the amplitude and/or the latency of responses across trials. One way of separating these two components is by performing a spectral decomposition analysis and examining the power and phase variability across trials within specific frequency bands. Below are results from the first stimulus interval and equivalent results were found in the second stimulus interval.

We first examined the mean power dynamics after stimulus presentation in four frequency bands: theta $(4-7 \mathrm{~Hz})$, alpha $(8-13 \mathrm{~Hz})$, beta $(14-20 \mathrm{~Hz})$, and low-gamma $(25-40 \mathrm{~Hz})$. This analysis revealed a typical initial broadband increase in power 100 ms after stimulus onset, which transitioned into a decrease in power $\sim 150 \mathrm{~ms}$ after stimulus onset (Fig. 7). ITPC analysis revealed an increase in phase coherence (i.e., phase alignment across trials) $100 \mathrm{~ms}$ after stimulus onset, which lasted until $\sim 300$ ms after stimulus onset.

\section{Variability quenching is associated with decreased power rather than increased ITPC}

Neural variability quenching may happen because of a decrease in power (i.e., less amplitude variability across trials) and/or an increase in phase coherence (i.e., less temporal variability across trials). Therefore, we examined the relationships across these measures in the 150-400 ms poststimulus time window when neural variability quenching was maximal (Fig. 3). Significant positive correlations were found between the magnitude of variability quenching and the power of theta-, alpha-, beta-, and low-gamma-frequency bands (Pearson's: $r_{(22)}>0.66, p<0.001$, Spearman's: $\left.r_{(22)}>0.65, p<0.001\right)$, indicating that individuals who exhibited larger variability quenching also exhibited weaker power in all four frequency bands. The relationship between variability quenching and ITPC was not significant for the alpha-, 

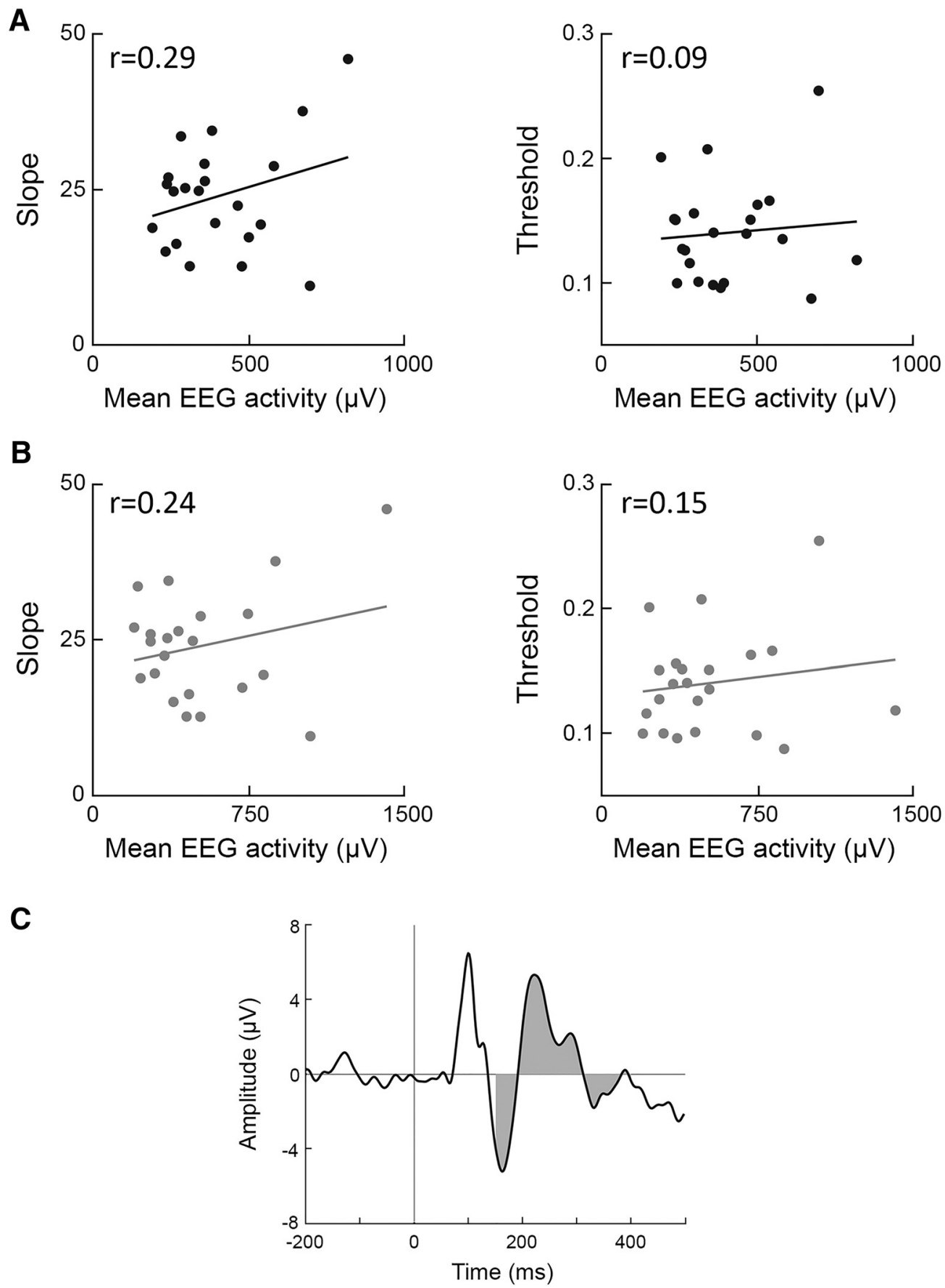

Figure 10. Mean EEG activity was not associated with perceptual measures. Shown are scatter plots demonstrating the relationship between mean EEG activity and psychometric function slope (left) or discrimination threshold (right) in the first $(\boldsymbol{A})$ or second $(\boldsymbol{B})$ stimulus interval. Each dot represents a single subject and Pearson's correlation coefficients are noted in each panel. $C$, Example demonstrating the calculation of the mean EEG response with the ERP of a single electrode in a single subject. Gray indicates area under the curve $150-400 \mathrm{~ms}$ after stimulus presentation.

beta-, and low-gamma-frequency bands (Pearson's: $r_{(22)}<0.16$, $p>0.24$; Spearman's: $\left.r_{(22)}<0.15, p>0.25\right)$, yet there was a significant correlation in the theta band when we used the Spearman's correlation coefficient (Pearson's: $r_{(22)}=0.28, p=0.1$; Spearman's: $\left.r_{(22)}=0.42, p<0.05\right)$. Together, these results suggest that individuals who quench more do so because of decreased power (i.e., lower amplitude variability across trials) rather than increased phase coherence (i.e., less temporal variability; Fig. 8).
Better perception is associated with decreased power rather than increased ITPC

Finally, we examined the relationship between the perceptual performance measures and spectral power or ITPC (Fig. 9). Significant negative correlations were found between the psychometric function slopes and the power in alpha-, beta-, and lowgamma-frequency bands (Pearson's: $r_{(22)}<-0.38, p<0.05$; Spearman's: $\left.r_{(22)}<-0.39, p<0.05\right)$ and a similar trend was found for the theta-frequency band (Pearson's: $r_{(22)}=-0.25$, 
$p=0.13$; Spearman's: $r_{(22)}=-0.29, p=$ $0.09)$. Significant positive correlations were found between the discrimination threshold and the power in alpha- and low-gamma-frequency bands (Pearson's: $r_{(22)}>0.35, p<0.05$; Spearman's: $r_{(22)}$ $>0.38, p<0.05)$ and similar trends in the theta- and beta-frequency bands (Pearson's: for theta and beta $r_{(22)}=0.32, p=$ 0.07; Spearman's: theta $r_{(22)}=0.3, p=$ 0.09 , beta $\left.r_{(22)}=0.35, p<0.05\right)$. ITPC measures were not correlated with either perceptual measure (Pearson's: $-0.07<$ $r<0.12, p>0.28$; Spearman's: $-0.09<$ $r<0.1, p>0.31$ ).

\section{Mean EEG activity was not associated with perceptual abilities}

We investigated whether there was a relationship between the two perceptual measures and mean EEG activity within the time window of quenching (i.e., $150-400$ ms after stimulus). Mean EEG activity was computed as the integral of the ERP (see Materials and Methods; Fig. 10C). Mean EEG activity was not significantly correlated with discrimination threshold nor with the psychometric function slope when examining responses from either the first (Pearson's: $r_{(22)}<0.28, p>0.1$; Spearman's: $\left.r_{(22)}<0.13, p>0.27\right)$ or second (Pearson's: $r_{(22)}<0.24, p>0.13$; Spearman's: $\left.r_{(22)}<0.12, p>0.29\right)$ stimulus interval (Fig. 10). This demonstrated that individual variability magnitudes rather than response amplitudes were significantly associated with perceptual performance measures.

\section{Alternative sources of trial-by- \\ trial variability}

To rule out potential confounds in the interpretation of our results, we examined whether individual differences in the variability of gaze position or quality of EEG recordings (as estimated by the variability of electrode offset across trials) could explain individual differences in neural variability measures. Gaze position variability and electrode offset variability across trials (see Materials and Methods) were not significantly correlated with magnitudes of variability quenching when examining either the first or second stimulus interval (Fig. 11). Finally, we also examined the goodness-offit of individual psychometric functions using the deviance measure and found that the deviances of all subjects were within an acceptable confidence range (i.e., between the $5^{\text {th }}$ and $95^{\text {th }}$ percentile of expected deviances distribution; see Materials and Methods). Most importantly, individual variability quenching was not significantly correlated with individual deviance measures (Fig. 11C). This means that potential between-subject differences in our ability to fit
A First stimulus interval

\section{Second stimulus interval}
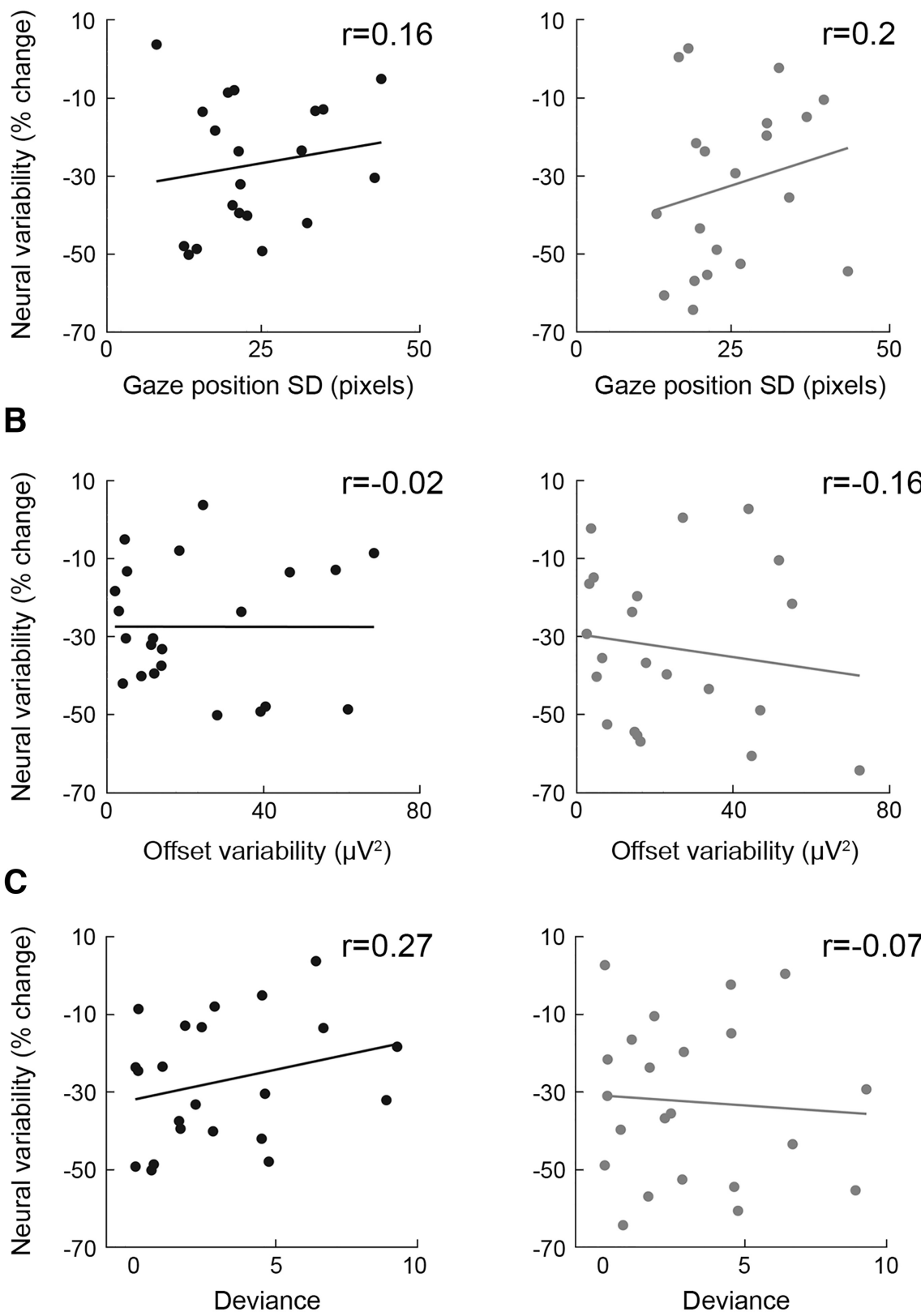

Figure 11. Individual magnitudes of variability quenching were not correlated with different sources of measurement noise. $A$, Correlation between variability quenching and gaze variability across trials in the first (left) and second (right) stimulus intervals. $\boldsymbol{B}$, Correlation between variability quenching and electrode offset variability across trials in the first (left) and second (right) stimulus intervals. C, Correlation between variability quenching and the goodness-of-fit of individual psychometric functions in the first (left) and second (right) stimulus intervals. Each point represents a single subject. Correlation coefficients are noted in each panel.

the psychometric function (i.e., goodness-of-fit) do not explain our results.

\section{Discussion}

Our results reveal that neural variability quenching differs across subjects in a manner that explains to a certain degree their individual perceptual abilities. Individuals who exhibited larger quenching had smaller contrast discrimination thresholds and steeper psychometric function slopes. These results were reproduced when examining neural responses from the first or 
second stimulus intervals, when examining the ICA components that best captured the visual responses (Fig. 4), and when quantifying quenching levels using three alternative measures (Figs. 3, 5).

Variability quenching measures explained perceptual abilities much better than absolute neural variability measures. Absolute neural variability after stimulus presentation was not significantly associated with perceptual performance, whereas neural variability before stimulus presentation was significantly correlated only with psychometric function slopes (Fig. 6). Nevertheless, a combination of large prestimulus neural variability and strong quenching seems to characterize individuals with superior perceptual performance.

Spectral analyses revealed that quenching intensities were strongly correlated with poststimulus reductions in the power of theta-, alpha-, beta- and low-gamma-frequency bands. Individuals who exhibited larger quenching intensities also exhibited larger reductions in poststimulus power (Fig. 8). In contrast, neural variability quenching did not correlate with the level of phase coherence across trials (ITPC). Furthermore, better perceptual performance was more strongly correlated with power reduction than with ITPC (Fig. 9). This reveals that larger variability quenching and better perceptual performance are associated with a decrease in the amplitude of broadband EEG oscillations after stimulus presentation rather than an increase in phase locking (i.e., timing of responses) across trials.

Together, these results suggest that the intensity of neural variability quenching may govern individual perceptual capabilities and that this phenomenon is generated by reducing the amplitude of broadband neural oscillations after stimulus presentation.

\section{Importance of reproducible neural responses across trials}

Previous electrophysiology and neuroimaging studies have revealed that trial-by-trial neural variability is reduced after stimulus presentation compared with the variability apparent beforehand. This robust phenomenon appears in responses of multiple brain areas when measured with intracellular (Monier et al., 2003; Finn et al., 2007), extracellular (Mitchell et al., 2007; Churchland et al., 2010; Hussar and Pasternak, 2010), fMRI (Schurger et al., 2010; Xue et al., 2010; He, 2013), MEG (Schurger et al., 2015), or ECOG (He and Zempel, 2013) recording techniques. This suggests that large moment-to-moment neural variability generated by ongoing spontaneous neural fluctuations is quenched by external neural input into the cortex. In other words, cortical networks seem to enforce more reproducible activity after receiving input about an external stimulus (Churchland et al., 2010).

Trials with more reproducible neural responses are often associated with faster and more accurate perception/behavior. For example, recordings in monkeys trained to attend to specific visual locations revealed that neural responses in visual system areas were less variable across trials when the stimulus was in the attended location (Mitchell et al., 2007; Cohen and Maunsell, 2009). This suggests that the beneficial effects of attention on behavioral speed and accuracy are associated with a decrease in neural variability. Furthermore, larger reductions in neural variability were apparent in trials in which a threshold-level stimulus was consciously perceived compared with trials where the stimulus was not (Schurger et al., 2010, 2015) and trial-by-trial variability was lower in trials in which a stimulus was successfully remembered $6 \mathrm{~h}$ later (Xue et al., 2010).
Although these studies present strong evidence in support of the hypothesis that larger reproducibility enables more accurate behavioral performance, previous studies did not examine how absolute and relative measures of neural variability may differ across individual animals/humans and potentially explain their individual behavioral capabilities.

\section{Neural variability in the prestimulus interval}

Although neural reproducibility seems to be important for perception, individuals with larger trial-by-trial variability in the prestimulus interval exhibited better perceptual performance measures as demonstrated by significant correlations with individual psychometric function slopes (Fig. 6). This seems surprising given the findings described above and the common intuition that larger variability should be detrimental for perception as outlined by signal detection theory principles (see section below).

With that said, several recent studies have suggested that larger temporal neural variability (e.g., the magnitude of ongoing fluctuations apparent in fMRI time courses) may be beneficial for cognitive performance (Garrett et al., 2013b) following the logic that variable neural systems may be able to encode a larger dynamic range of stimuli and transition across "brain states" with better efficiency (Deco et al., 2011; Mišić et al., 2011). This view is based on reports of individuals who exhibit larger fMRI time course variability and perform cognitive tasks such as perceptual matching, attentional cueing, and delayed match to sample more quickly and consistently (Garrett et al., 2013a).

Our results offer a potential way of bridging these conflicting reports, which have measured neural variability using different analysis techniques and mostly focused on different types of variability: variability of stimulus-evoked responses across trials (Schurger et al., 2010; Xue et al., 2010) and ongoing variability over time regardless of stimulus/task presentation (Garrett et al., 2011, 2014). By separating prestimulus and poststimulus variability and demonstrating that their ratio enables the strongest prediction of individual perceptual abilities, our results highlight the importance of relative rather than absolute variability measures for explaining, at least in part, individual perceptual performance levels. This is consistent with a recent MEG study reporting larger variability quenching across trials in which a threshold-level stimulus was accurately detected as well as larger ongoing/baseline variability in healthy adults compared with unconscious patients (Schurger et al., 2015).

\section{Signal detection theory}

Signal detection theory describes several factors that are commonly thought to affect the ability of a system to detect the presence of a stimulus (Green and Swets, 1966); two basic factors are the intensity of the stimulus and the amount of internal noise within the system. When the signal intensity is strong and the amount of internal noise is low it is easy to detect the presence of the stimulus. Trial-by-trial neural variability is a measure of the internal noise of a sensory neural system. According to signal detection theory, minimizing such variability would enable more accurate detection of weaker stimuli.

Previous studies have estimated internal noise using behavioral measures including the equivalent noise model (Pelli and Farell, 1999), the double-pass method (Burgess and Colborne, 1988; Neri, 2010), or by examining the slope of the psychometric function (Buss et al., 2009; Jones et al., 2014). Such studies have reported that individuals with lower levels of internal noise exhibit better perceptual performance (Legge et al., 1987; Pardhan, 
2004; Aihara et al., 2008) and have inferred that internal noise limits the perceptual abilities of an individual.

In the current study, we assessed internal noise in individual subjects by quantifying EEG trial-by-trial variability. In contrast to the psychophysics studies described above, our results suggest that it is the relative (rather than absolute) level of internal noise (i.e., internal noise quenching) that governs performance differences across individual subjects.

\section{What drives neural variability quenching?}

Two distinct sources of variability can generate trial-by-trial EEG variability: (1) variability in the amplitude of the responses across trials and (2) variability in the phase (i.e., timing/latency) of the responses across trials. We used spectral analyses to disentangle the contributions of amplitude and phase changes to variability quenching. These analyses revealed that variability quenching was strongly associated with a decrease in broadband EEG power, but not with an increase in ITPC (Fig. 8). This suggests that neural variability quenching may be the outcome of decreased theta-, alpha-, beta-, and low-gamma-band power that appears 150-200 ms after stimulus presentation. Furthermore, a follow-up analysis revealed that individuals with weaker power (rather than stronger ITPC) exhibited better perceptual performance (Fig. 9). This suggests that the better cortical reproducibility after stimulus presentation (Churchland et al., 2010) is mostly enforced by reducing broadband oscillatory power.

Reduction in alpha/beta-band power that appears after stimulus presentation is a widely reported phenomenon that has been named event-related desynchronization (ERD) (Pfurtscheller and Lopes da Silva, 1999; Klimesch, 2012). ERD responses are spatially selective to the activated cortical location (Pfurtscheller and Lopes da Silva, 1999; Nikouline et al., 2000), are larger on trials where subjects allocate attention to the stimulus (Foxe and Snyder, 2011), increase with stimulus amplitude (Stancák et al., 2003), and are larger on trials in which stimuli are consciously perceived (Babiloni et al., 2006). These findings support a general claim that lower alpha power (i.e., larger ERD) reflects a higher level of cortical activation (Pfurtscheller, 2006). To date, the relationship between neural variability quenching and ERD has not been examined and the two measures have mostly been reported and discussed in separate fields of research. Our results suggest that there is likely to be an intimate relationship between the two, which seems to characterize complementary aspects (activation level and reproducibility) of stimulus-evoked cortical responses that differ across individuals with different perceptual abilities.

\section{Dealing with measurement noise}

When quantifying differences in trial-by-trial neural variability across subjects, it is essential to minimize the contribution of nonneural sources of variability that may also differ across subjects. To address this concern, we carefully cleaned the data using automated procedures for identifying and removing trials with eye blinks, muscle contractions, and saccades. In addition, we performed an ICA analysis and selected the spatiotemporal components that best captured the visual responses in our experiment, thereby excluding all ICA components that may have contained variability that was not necessarily associated with neural activity (Fig. 4). Equivalent findings were apparent when using the ICA analysis. This demonstrates the robustness of the findings across multiple processing and cleaning techniques, ruling out potential contributions of measurement noise.

\section{Conclusions}

Until recently, measures of neural variability within single subjects were mostly ignored by the scientific community and considered to be meaningless "noise." Accumulating research is demonstrating that measures of neural variability are useful for explaining behavioral variability across trials and differences in perceptual capabilities across individuals. Important outstanding questions for future research include that following. Are withinsubject neural variability measures (e.g., variability quenching) reliable over time and across tasks? To what degree is neural variability under behavioral control of an individual through mechanisms of attention and neuromodulation? How can neural variability be altered to determine causal relationships with behavioral measures? Answering these questions and others by examining absolute and relative variability measures in perceptual/ cognitive tasks across multiple sensory modalities and across different patient populations are likely to reveal rich sources of information for accurately characterizing individual perceptual and cognitive abilities and clinical states (Dinstein et al., 2015).

\section{References}

Aihara T, Kitajo K, Nozaki D, Yamamoto Y (2008) Internal noise determines external stochastic resonance in visual perception. Vision Res 48 : 1569-1573. CrossRef Medline

Arieli A, Sterkin A, Grinvald A, Aertsen A (1996) Dynamics of ongoing activity: explanation of the large variability in evoked cortical responses. Science 273:1868-1871. CrossRef Medline

Babiloni C, Vecchio F, Bultrini A, Luca Romani G, Rossini PM (2006) Preand poststimulus alpha rhythms are related to conscious visual perception: a high-resolution EEG study. Cereb Cortex 16:1690-1700. Medline

Brainard DH (1997) The psychophysics toolbox. Spat Vis 10:433-436. CrossRef Medline

Burgess AE, Colborne B (1988) Visual signal detection. IV. Observer inconsistency. J Opt Soc Am A 5:617-627. CrossRef Medline

Buss E, Hall JW 3rd, Grose JH (2009) Psychometric functions for pure tone intensity discrimination: slope differences in school-aged children and adults. J Acoust Soc Am 125:1050-1058. CrossRef Medline

Carandini M (2004) Amplification of trial-to-trial response variability by neurons in visual cortex. PLoS Biol 2:E264. CrossRef Medline

Churchland AK, Kiani R, Chaudhuri R, Wang XJ, Pouget A, Shadlen MN (2011) Variance as a signature of neural computations during decision making. Neuron 69:818-831. CrossRef Medline

Churchland MM et al. (2010) Stimulus onset quenches neural variability: a widespread cortical phenomenon. Nat Neurosci 13:369-378. CrossRef Medline

Cohen MR, Maunsell JH (2009) Attention improves performance primarily by reducing interneuronal correlations. Nat Neurosci 12:1594-1600. CrossRef Medline

Deco G, Jirsa VK, McIntosh AR (2011) Emerging concepts for the dynamical organization of resting-state activity in the brain. Nat Rev Neurosci 12:43-56. CrossRef Medline

Delorme A, Makeig S (2004) EEGLAB: An open source toolbox for analysis of single-trial EEG dynamics including independent component analysis. J Neurosci Methods 134:9-21. CrossRef Medline

Dienes Z (2014) Using Bayes to get the most out of non-significant results. Front Psychol 5:781. CrossRef Medline

Dinstein I, Heeger DJ, Behrmann M (2015) Neural variability: friend or foe? Trends Cogn Sci 19:322-328. CrossRef Medline

Finn IM, Priebe NJ, Ferster D (2007) The emergence of contrast-invariant orientation tuning in simple cells of cat visual cortex. Neuron 54:137-152. CrossRef Medline

Foxe JJ, Snyder AC (2011) The role of alpha-band brain oscillations as a sensory suppression mechanism during selective attention. Front Psychol 2:154. CrossRef Medline

Garrett DD, Kovacevic N, McIntosh AR, Grady CL (2011) The importance of being variable. J Neurosci 31:4496-4503. CrossRef Medline

Garrett DD, Kovacevic N, McIntosh AR, Grady CL (2013a) The modulation of BOLD variability between cognitive states varies by age and processing speed. Cereb Cortex 23:684-693. CrossRef Medline 
Garrett DD, Samanez-Larkin GR, MacDonald SW, Lindenberger U, McIntosh AR, Grady CL (2013b) Moment-to-moment brain signal variability: a next frontier in human brain mapping? Neurosci Biobehav Rev 37:610-624. CrossRef Medline

Garrett DD, McIntosh AR, Grady CL (2014) Brain signal variability is parametrically modifiable. Cereb Cortex 24:2931-2940. Medline

Goris RL, Movshon JA, Simoncelli EP (2014) Partitioning neuronal variability. Nat Neurosci 17:858-865. CrossRef Medline

Green DM, Swets JA (1966) Signal detection theory and psychophysics. New York: John Wiley.

Hawkey DJ, Amitay S, Moore DR (2004) Early and rapid perceptual learning. Nat Neurosci 7:1055-1056. CrossRef Medline

He BJ (2013) Spontaneous and task-evoked brain activity negatively interact. J Neurosci 33:4672-4682. CrossRef Medline

He BJ, Zempel JM (2013) Average is optimal: an inverted-U relationship between trial-to-trial brain activity and behavioral performance. PLoS Comput Biol 9:e1003348. CrossRef Medline

Hussar C, Pasternak T (2010) Trial-to-trial variability of the prefrontal neurons reveals the nature of their engagement in a motion discrimination task. Proc Natl Acad Sci U S A 107:21842-21847. CrossRef Medline

Jones PR, Moore DR, Shub DE, Amitay S (2014) Learning to detect a tone in unpredictable noise. J Acoust Soc Am 135:EL128-EL133. CrossRef Medline

Wagenmakers EJ, Verhagen J, Ly A (2016) How to quantify the evidence for the absence of a correlation. Behav Res Methods 48:413-426. CrossRef Medline

Kappenman ES, Luck SJ (2010) The effects of electrode impedance on data quality and statistical significance in ERP recordings. Psychophysiology 47:888-904. CrossRef Medline

Klimesch W (2012) $\alpha$-band oscillations, attention, and controlled access to stored information. Trends Cogn Sci 16:606-617. CrossRef Medline

Ledberg A, Montagnini A, Coppola R, Bressler SL (2012) Reduced variability of ongoing and evoked cortical activity leads to improved behavioral performance. PLoS One 7:e43166. CrossRef Medline

Legge GE, Kersten D, Burgess AE (1987) Contrast discrimination in noise. J Opt Soc Am A 4:391-404. CrossRef Medline

Levitt H (1970) Transformed up-down methods in psychoacoustics. J Acoust Soc Am 33:467-477.

Makeig S, Jung TP, Bell AJ, Ghahremani D, Sejnowski TJ (1997) Blind separation of auditory event-related brain responses into independent components. Proc Natl Acad Sci U S A 94:10979-10984. CrossRef Medline

Mišić B, Vakorin VA, Paus T, McIntosh AR (2011) Functional embedding predicts the variability of neural activity. Front Syst Neurosci 5:90. CrossRef Medline

Mitchell JF, Sundberg KA, Reynolds JH (2007) Differential attention- dependent response modulation across cell classes in macaque visual area V4. Neuron 55:131-141. CrossRef Medline

Monier C, Chavane F, Baudot P, Graham LJ, Frégnac Y (2003) Orientation and direction selectivity of synaptic inputs in visual cortical neurons: a diversity of combinations produces spike tuning. Neuron 37:663-680. CrossRef Medline

Neri P (2010) How inherently noisy is human sensory processing? Psychon Bull Rev 17:802-808. CrossRef Medline

Nikouline VV, Linkenkaer-Hansen K, Wikström H, Kesäniemi M, Antonova EV, Ilmoniemi RJ, Huttunen J (2000) Dynamics of mu-rhythm suppression caused by median nerve stimulation: a magnetoencephalographic study in human subjects. Neurosci Lett 294:163-166. CrossRef Medline

Pardhan S (2004) Contrast sensitivity loss with aging: sampling efficiency and equivalent noise at different spatial frequencies. J Opt Soc Am A 21:169-175. CrossRef

Pelli DG, Farell B (1999) Why use noise? J Opt Soc Am A 16:647. CrossRef Pfurtscheller G (2006) The cortical activation model (CAM). Prog Brain Res 159:19-27. CrossRef Medline

Pfurtscheller G, Lopes da Silva FH (1999) Event-related EEG/MEG synchronization and desynchronization: basic principles. Clin Neurophysiol 110:1842-1857. CrossRef Medline

Prins N, Kingdom FAA (2009) Palamedes: Matlab routines for analyzing psychophysical data. Available from: www.palamedestoolbox.org.

Qi XL, Constantinidis C (2012) Variability of prefrontal neuronal discharges before and after training in a working memory task. PLoS One 7:e41053. CrossRef Medline

Schurger A, Pereira F, Treisman A, Cohen JD (2010) Reproducibility distinguishes conscious from nonconscious neural representations. Science 327:97-99. CrossRef Medline

Schurger A, Sarigiannidis I, Naccache L, Sitt JD, Dehaene S (2015) Cortical activity is more stable when sensory stimuli are consciously perceived. Proc Natl Acad Sci U S A 112:E2083-E2092. CrossRef Medline

Shadlen MN, Newsome WT (1998) The variable discharge of cortical neurons: implications for connectivity, computation, and information coding. J Neurosci 18:3870-3896. Medline

Stancák A, Svoboda J, Rachmanová R, Vrána J, Králík J, Tintera J (2003) Desynchronization of cortical rhythms following cutaneous stimulation: effects of stimulus repetition and intensity, and of the size of corpus callosum. Clin Neurophysiol 114:1936-1947. CrossRef Medline

Wichmann FA, Hill NJ (2001) The psychometric function: I. Fitting, sampling, and goodness of fit. Percept Psychophys 63:1293-1313. CrossRef Medline

Xue G, Dong Q, Chen C, Lu Z, Mumford JA, Poldrack RA (2010) Greater neural pattern similarity across repetitions is associated with better memory. Science 330:97-101. CrossRef Medline 